\title{
A Novel Battery State of Health Estimation Method Based on Outlier Detection Algorithm
}

\author{
Chang-hao Piao*, Zi-hao Hu*, Ling Su** and Jian-fei Zhao ${ }^{\dagger}$
}

\begin{abstract}
A novel battery SOH estimation algorithm based on outlier detection has been presented. The Battery state of health $(\mathrm{SOH})$ is one of the most important parameters that describes the usability state of the power battery system. Firstly, a battery system model with lifetime fading characteristic was established, and the battery characteristic parameters were acquired from the lifetime fading process. Then, the outlier detection method based on angular distribution was used to identify the outliers among the battery behaviors. Lastly, the functional relationship between battery $\mathrm{SOH}$ and the outlier distribution was obtained by polynomial fitting method. The experimental results show that the algorithm can identify the outliers accurately, and the absolute error between the $\mathrm{SOH}$ estimation value and true value is less than $3 \%$.
\end{abstract}

Keywords: State of health (SOH), Outlier detection, Angular distribution, Fitting function

\section{Introduction}

Environment pollution, energy shortage and large demand of vehicle make people to seek new technological breakthrough in vehicle power system field. The electric vehicle is an effective way to solve the above problems. As the main power resource of the electric vehicle, the battery system and its states have a significant effect on the vehicle's power performance, economy and security [1-4].

Therefore, the study on battery system state estimations has become one of the core technologies.

Battery state estimations consist of state of charge (SOC) estimation and $\mathrm{SOH}$ estimation et.al. At present, the estimation methods for battery SOC have become more and more mature. While, the estimation methods for battery $\mathrm{SOH}$ are still in research stage, and rarely get into practical application. As one of the key tasks of battery state estimations, the estimation methods for battery $\mathrm{SOH}$ have obtained more and more attentions among the domestic and overseas researchers.

So far, the common vehicle power battery SOH estimation methods are implemented by battery intrinsic parameters estimation and identification. The concept of battery $\mathrm{SOH}$ was proposed firstly in 1998 [5]. Since then, the research focus was gradually turned to the investigation of rated capacity variation characteristics and internal resistance variation characteristics [6,7]. Gregory [8] put forward a battery $\mathrm{SOH}$ estimation algorithm based on Double

$\dagger$ Corresponding Author: Institution of Pattern Recognition and Application, Chongqing University of Posts and Telecommunications China. (1271020776@qq.com)

* Institution of Pattern Recognition and Application, Chongqing University of Posts and Telecommunications Chongqing, China

** Chongqing Changan New Energy Automobile CO., LTD., Chongqing China.

Received: April 28, 2015; Accepted: June 13, 2016
Extended Kalman Filter (DEKF) method. Dai Haifeng and Wei Xuezhe [9] made further studies on the DEKF method. Nakamur [10] presented an algorithm for battery internal state estimation through adaptive digital filter, and got the $\mathrm{SOH}$ value by using recursive least square algorithm to estimate the internal resistance. Song [11] proposed a battery $\mathrm{SOH}$ estimation algorithm using double sliding mode observer to estimate capacity and internal resistance. The above battery $\mathrm{SOH}$ estimation methods are all implemented based on the battery rated capacity or internal resistance estimation. But it is difficult to obtain the accurate values of battery rated capacity or internal resistance in reality. So the estimation error is relatively large.

Outlier detection is one of the most important aspects in data mining field. It has been used in various fields such as credit card fraud checking, network intrusion detection and weather forecast, etc [12]. The existence of outliers has a significant impact on the analysis of time series, such as the autocorrelation and partial correlation analysis, parameter estimation, result prediction, and even pattern identification. At present, there is still no research using outlier detection to evaluate the battery $\mathrm{SOH}$ in domestic and abroad. While researchers have developed lots of different outlier detection algorithms. Among these algorithms, the outlier detection algorithm based on angular distribution performed better in analysis of battery lifetime data because of following characteristics, i.e. high dimension, parameter shading and big data, etc.

Aiming at the battery lifetime data characteristics, a novel battery $\mathrm{SOH}$ estimation method using the outlier detection algorithm based on angular distribution is presented. The battery characteristic parameters such as battery voltage, current, temperature, SOC are form the battery lifetime data set. Then the outlier detection algorithm is used to identify the states of the outlier sets in the battery lifetime 
data sets. Lastly, the fitting function between the outlier state and battery $\mathrm{SOH}$ is constructed to implement the effective battery $\mathrm{SOH}$ estimation.

\section{Theory of Outlier Detection Algorithm}

The outlier detection method can find out the outliers or the isolate points through analysing the information characteristics. This mechanism can be applied to solve the outlier identification problem during the battery lifetime aging process. At present, the outlier detection algorithms are mainly divided into following types: statistics-based, distance-based, density-based and angular-distributionbased [13]. The first three algorithms are maturely studied, but have poor practicability in high-dimensional data. So, in this article, the angular-distribution-based outlier detection algorithm is adopted to analyse the battery characteristics parameters.

The concept of calculating the abnormal degree of each point in data set by angular distribution method was given [14]. Assuming a simple two-dimensional data set, the point $\mathrm{O}$ is a divorced point deviating from group and the point $B$ is in the interior of the group, other points are between point $\mathrm{O}$ and point $\mathrm{B}$. Where the angular range formed by point $\mathrm{O}$ and two other points in the data set is limited, its fluctuation is relatively small. While the angular range formed by point $\mathrm{B}$ and two other points in the data set is wide, its fluctuation is relatively large. The angular fluctuation of the boundary points is between the two above. Therefore, the angle variance can be used to measuring the abnormal degree of each point, and thus distinguishing the above three types of points.

Based on the above theory, Pham et al. proposed the concept of angle distribution abnormal factor [15], and the specific definition is as follows:

Assuming a sample data set $S \subseteq R^{d},|S|=n$, a sample point $p \in S$, and a pair of sample points $a, b \in S \backslash\{p\}$. $\Theta_{a p b}$ are selected randomly to represent the angle value between different vectors $a-p$ and $b-p$. Thus the variance of the whole $\Theta_{a p b}$ is the angle distribution abnormal factor $\operatorname{VOA}(p)$.

$$
\operatorname{VOA}(p)=\operatorname{Var}\left[\Theta_{a p b}\right]=M O A_{2}(p)-\left(M O A_{1}(p)\right)^{2}
$$

where,

$$
\begin{gathered}
\sum_{1}(p)=2 \frac{\sum_{\substack{a, b \in S \backslash\{p\}, a \neq b}} \Theta_{a p b}}{(n-1)(n-2)} \\
\sum_{\substack{a, b \in S \backslash\{p\}, a}} \Theta^{2}{ }_{a p b} \\
(n-1)(n-2)
\end{gathered}
$$

Kriegel's research indicates that if a point has smaller
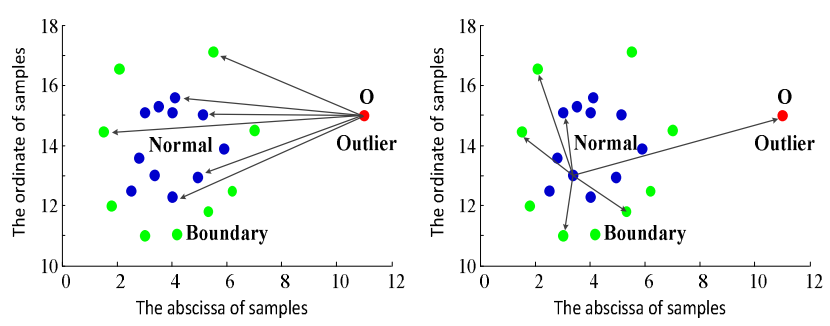

Fig. 1. Outlier distribution

angle variance $\operatorname{VOA}(p)$, it will be more likely to be an outlier point. Therefore, the abnormal degree of each point could be obtained by its angle variance, and the data set was distinguished to normal point, boundary point and abnormal point. However, at present there is not a good way to define the threshold, we can only select the best VOA_1, VOA_2 by experiments to ensure the ability to identify the outliers [16]. Outlier distribution is as shown in Fig. 1.

\section{Analysis of Battery SOH Characteristics Parameters and Lifetime Model}

\subsection{Analysis of battery SOH characteristics para- meters}

The power battery $\mathrm{SOH}$ can be reflected by the characteristic parameters of the battery itself. Therefore, the battery $\mathrm{SOH}$ can be evaluated by analysing the joint changes of several internal characteristic parameters. Through the present studies, it is known that there is a certain relationship between battery $\mathrm{SOH}$ and the battery characteristics parameters, i.e. voltage, current, temperature, SOC, internal resistance and rated capacity. Among the above parameters, the voltage, current and temperature are relatively easy to be measured, and the method of battery SOC estimation is more mature. However, the rated capacity and internal resistance measurement are difficult, and the relative error is larger. So, in this article, the changes of the following four parameters, i.e. voltage, current, temperature, and SOC, are selected as the battery $\mathrm{SOH}$ judgment basis.

Considering the electrochemical properties of the battery, the rated capacity and internal resistance are the most immediate characteristic parameters that reflect the battery $\mathrm{SOH}$ (especially the rated capacity) [17]. The $\mathrm{SOH}$ decrement of a battery cell is mostly caused by the battery aging and degradation, namely, durability problems. That means with the using or storing of the battery cells, the battery capacity would decrease and the resistance would increase. Thus the SOH of battery cells worsen [17]. But the process of estimating and measuring the rated capacity and internal resistance is complex. In order to make the SOH estimation algorithm be simple and feasible, a battery lifetime model is established, which takes the temperature 


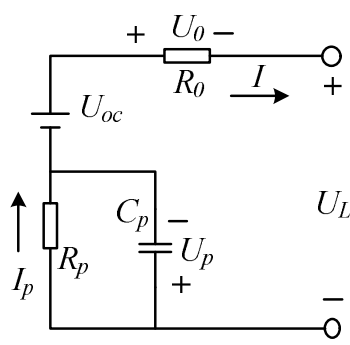

Fig. 2. Equivalent circuit model of Thevenin

Note: ideal voltage source $U_{o c}$ is the open circuit voltage of battery, $U_{L}$ is the battery terminal voltage, $R_{0}$ is the internal resistant

and the total amount of battery charge-discharge as the variables. And the battery lifetime model was integrated with the battery system model and hybrid vehicle model to construct a battery system simulation platform with lifetime attenuation characteristics.

\subsection{Car battery model}

Thevenin model is widely used at present, which considering the capacitance characteristics of the battery, and can also describe the electrochemical properties of cell accurately. The model using $\mathrm{RC}$ parallel network which consists of polarization capacity $C_{p}$ and polarization resistance $R_{p}$ can predict the battery polarization effect in cell load response process, simulating the dynamic characteristics of battery polarization in the process of generation and elimination. Equivalent circuit model is shown in Fig. 2.

The analysis shows that the establishment process of current battery model, which have not taken the battery aging and life attenuation into account. That is to say in the cell equivalent circuit model, the battery parameters such as resistance, capacity and so on are all got by empirical methods. However, their values are fixed in the process of model runs and it cannot describe the attenuation characteristics of battery life.

\subsection{Battery lifetime model}

Arrhenius equation describes the acceleration effect of the temperature stress on the battery lifetime. Based on the above theory, paper [18] built a two-factor lifetime model which takes Ah-throughput and temperature as variables. In this article, the charge-discharge rate multiply time to obtain a battery lifetime model that take Ah-throughput as independent variable, then taking temperature and chargedischarge rate is as accelerated stress. The study is based on Thevenin battery equivalent circuit model, considering the effects of charge-discharge rate and temperature on battery life, and establishing battery life model with growth of internal resistance and fade of battery capacity fade. Combining the battery life model with Thevenin battery
Table 1. Battery lifetime model and coefficient table ${ }^{[18]}$

\begin{tabular}{c|c|c|c|c}
\hline C_rate & $\mathrm{C} / 2$ & $2 \mathrm{C}$ & $6 \mathrm{C}$ & $10 \mathrm{C}$ \\
\hline $\mathrm{B}$ & 31630 & 21681 & 12934 & 15512 \\
\hline $\mathrm{z}$ & 0.552 & 0.554 & 0.56 & 0.56 \\
\hline
\end{tabular}

Note: on the above formula, $Q_{\text {loss }}$ is the percentage of battery capacity fading, $t$ is the running time, and $T$ is the test temperature (absolute temperature), DOD is the depth of the discharge, and C_rate is charge-discharge rate.

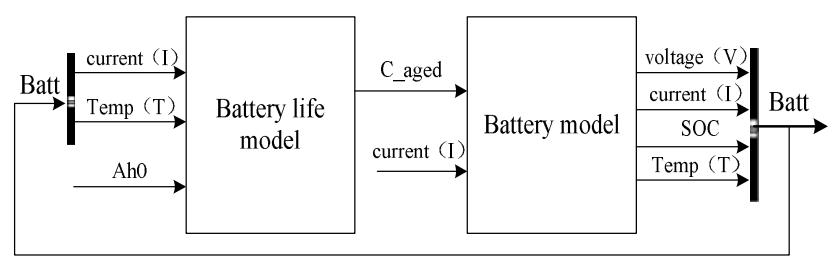

Fig. 3. The relation diagram of battery lifetime model with battery model

equivalent circuit model is to constitute a battery life model with attenuation characteristics. And then use it as a basis for this study.

$$
\begin{aligned}
& Q_{\text {loss }}=B \cdot \exp \left[\frac{-31700+370.3 \times C_{\text {rate }}}{R T}\right](A h)^{z} \\
& A h=A h_{t_{0}}+\int_{t_{0}}^{t}|i| d(t)
\end{aligned}
$$

The coefficients of B and $\mathrm{z}$ in battery life models are just a discrete value corresponding to specific charge-discharge rate, but the charge-discharge rate of the battery under practical conditions is a continuous gradient. Considering the current of the battery system was gradual when established the battery life model, linear interpolation method was used to obtain the parameter $\mathrm{B}$ and $\mathrm{z}$ values under continuous discharge rate condition.

\subsection{Battery system simulation model with lifetime fading characteristics}

The literature [19] constructed a vehicle battery management system software platform in-loop, which included the battery system model, vehicle controller model, driver model, driving cycle model, powertrain model, vehicle dynamics model and wheel models, etc. The software platform can realize all the functions of the battery management system under vehicle condition and also can make a real-time monitoring and control of the power lithium battery pack. The battery lifetime model is added to the vehicle battery management system software platform in-loop. So, a battery system model with lifetime fading characteristics is constructed in this paper. The relation diagram of the battery lifetime model and battery system model is shown in Fig. 3. And the improved battery system model with lifetime fading characteristics is shown in Fig. 4. 


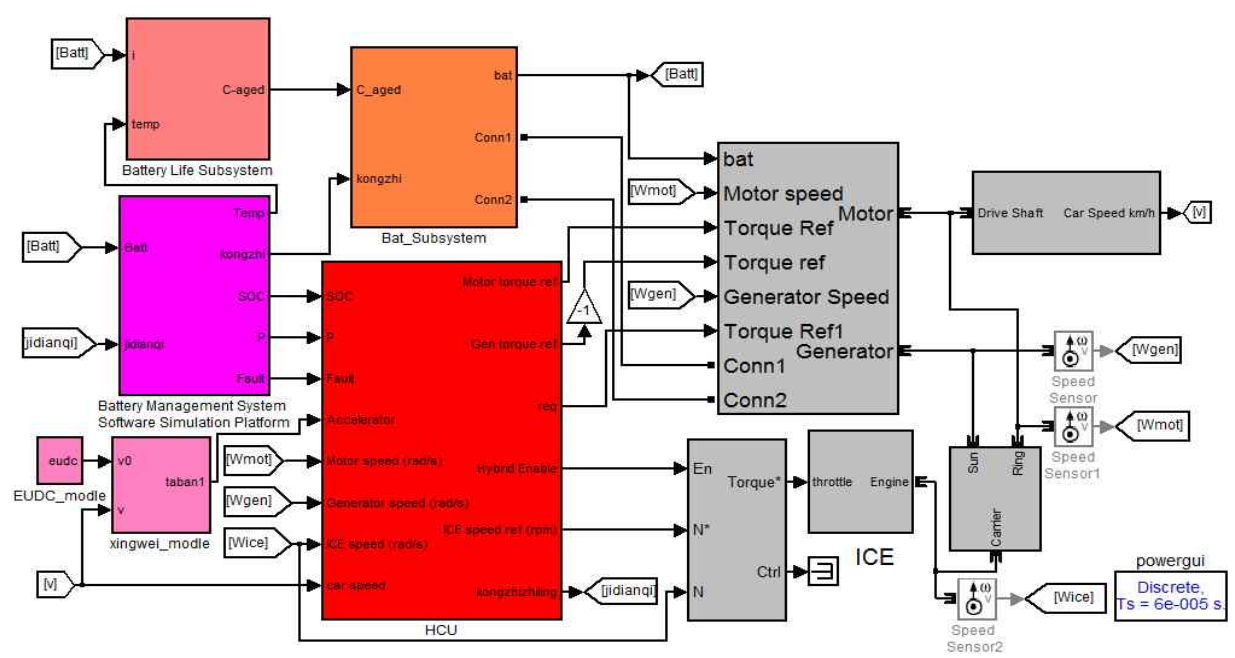

Fig. 4. The battery system simulation model with lifetime fading characteristics

\section{The Battery SOH Estimation Algorithm Based on Outlier Detection}

\subsection{The basic idea of the algorithm}

In this article, the proposed battery $\mathrm{SOH}$ estimation algorithm based on outlier detection could be described as follows:

1) Firstly, with the integration of hybrid vehicle model, a power battery model with lifetime fading characteristics was built to set up the battery system simulation platform.

2) Based on the above simulation platform, the characteristic parameters that can represent the battery system performance degradation are obtained during the process of battery lifetime fading. Then, the outlier detection algorithm based on angular distribution was built for the test data set.

3) After normalizing and pre-processing the test data set, the outlier detection algorithm based on angular distribution is used to identify the outliers in the test data set. Then, calculate the outlier state of the characteristic parameters that can represent the battery system performance degradation during the process of battery lifetime fading.

4) According to the above outliers state of the characteristic parameters, set up the relation model or fitting equation between the outliers state and the battery $\mathrm{SOH}$.

5) Finally, collect new characteristic parameters and get the outliers state. Then calculate the battery $\mathrm{SOH}$ value by the fitting equation.

\subsection{Algorithm detailed processes}

The detailed processes of the proposed battery $\mathrm{SOH}$ estimation algorithm based on outlier detection is shown in

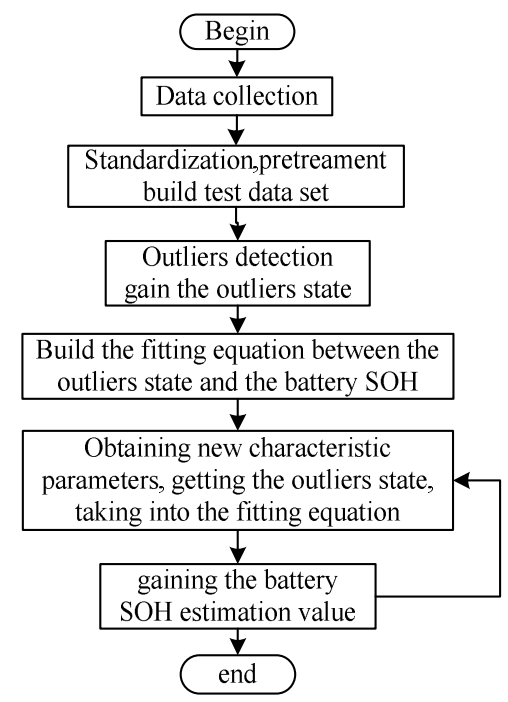

Fig. 5. The proposed battery SOH estimation method flow diagram

Fig. 6.

The specific processes of the algorithm are described as follows:

Input: the battery characteristics parameters data set $\mathrm{X}$

Output: the outlier set $\mathrm{O}$, and the battery $\mathrm{SOH}$ estimation value

1) System initialization, get the 4-dimensions and ngroups battery life data set $\mathrm{X}$ (voltage, current, temperature and SOC), and pre-treatment the test data set X.

2) Generate the initial test data set $X(m) \quad(\mathrm{m}=30)$. Use Eqs. (1), (2) and (3), to analyze each element in the set, and calculate the $V O A$ value of each element of characteristic parameter $X(m)$.

3) According to the $V O A$ value of each element, with the normal threshold and the outlier threshold, get the 


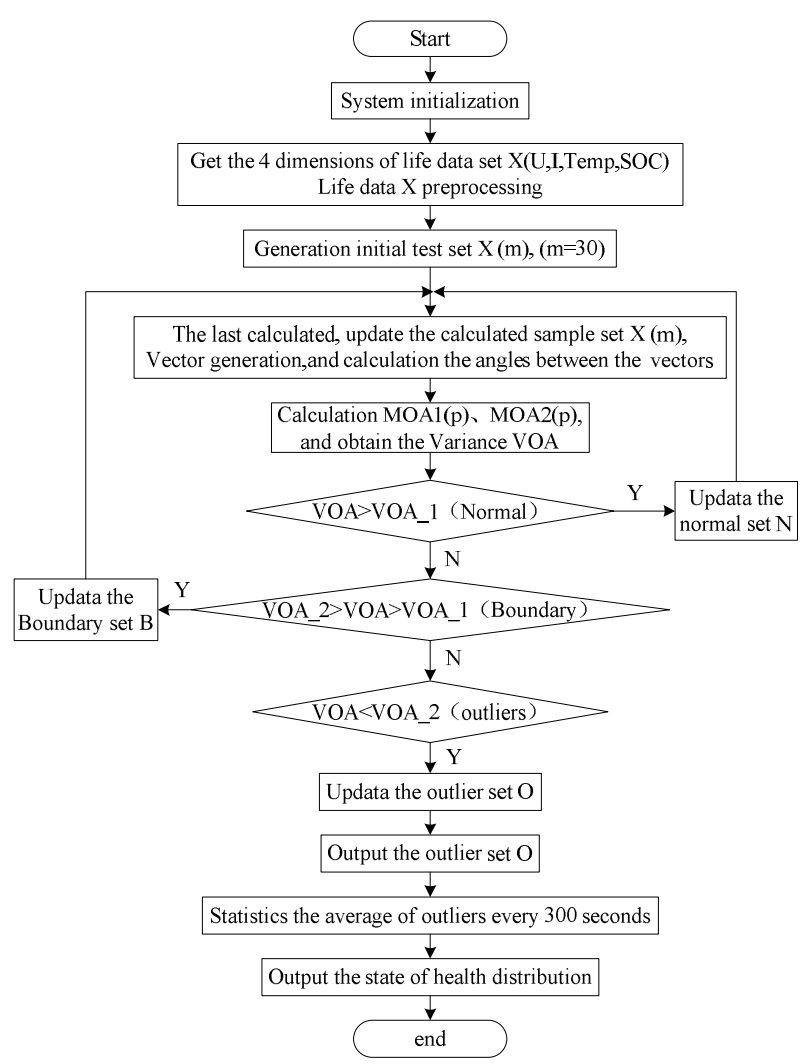

Fig. 6. The detailed process of the battery SOH estimation algorithm based on outlier detection

normal data set, boundary data set and outlier data set.

4) After the last calculation, get the latest data element $X(i)$, normal set and border set to update the calculation sample set. Use Eqs. (1), (2) and (3)to analyze $X(i)$ and obtain the outlier factor $V O A$ of $X(i)$.Compared the $V O A$ with the normal threshold and outlier threshold, if $X(i)$ is an outlier, $X(i)$ will be added to the outlier set and output at the same time. Otherwise, $X(i)$ will be added to the normal set or border set. The normal set and border set are updated by FIFO mode.

5) Repeat step 4) until the test data up to 3000 groups, and output the outlier set $\mathrm{O}$.

6) Get the latest data element $X(i)$, and repeat the above steps 2) 5) until all data detection complete under the standard working conditions. Calculate the average value of battery parameters outlier numbers every 300 seconds.

7) Finally, according to the final set $O$, conclude the battery $\mathrm{SOH}$ at present by the fitting equation.

\subsection{Algorithm implementation}

The step 2) above is the core of the whole algorithm, the value of $V O A$ can be calculated accurately and quickly by step 2). Assume that the input of step 2) consists of the latest data $X(i)$, normal set and boundary set, which is a data flow pattern calculation set, and the output is the $V O A$ value of $X(i)$. The specific implementation steps are as follows:

(1) Set the latest data element $X(i)$ as the vertex $p$;

(2) Get an arbitrary point $a$, which is inequality with point $p$ in the data set;

(3) Get another point $b$, which is inequality with point $p$ and point $a$, and find out all point $b$ in the data set;

(4) Calculate all the vector angles $\Theta_{a p b}$ generated by vector $\mathrm{p}-\mathrm{a}$ and vector $\mathrm{p}-\mathrm{b}$;

(5) Store the obtained $\Theta_{a p b}$ to the angle set $\Theta[i]$ respectively;

6) If there still exists untreated point $a$ in the data set, return to step (2);

(7) To deal with the $\Theta[i]$ by Eqs. (1), (2) and (3), obtain the $V O A$ value of each element $X[i]$.

\subsection{The sensitivity analysis of algorithm}

The sensitivity analysis of proposed method should be required, because of the influence on model in the process of data collection, such as systematic error, measurement noise, accidental error and so on. The simulation takes 20000 samples with 26 outliers(SOH is $92 \%$ according the fitting function) in $4 * \mathrm{ECE}+\mathrm{EUDC}$ condition and different intensity of noise into model. The definition of unbearable noise intensity in the sensitivity analysis of proposed method is that it leads to $5 \%$ fluctuation of the error (i.e. $87 \% \mathrm{SOH}$ to $97 \% \mathrm{SOH}$ ) for the estimate results of the proposed algorithm. And the result is shown in Table 2 . Analysis of the results known, the algorithm will be invalid if Gaussian white noise intensity is more than 8 .
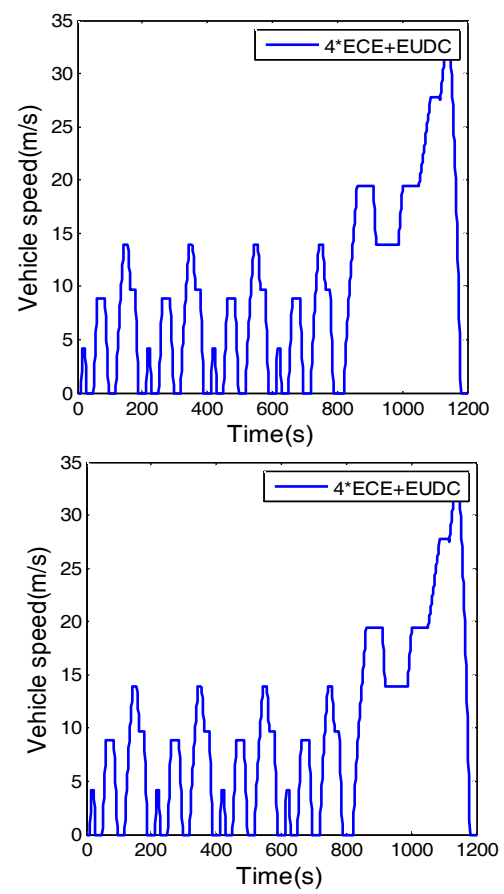

Fig. 7. 4*ECE+EUDC and FTP75 conditions 
Table 2. The outlier test results and statistics in $4 \times \mathrm{ECE}+$ EUDC condition

\begin{tabular}{ccccccccc}
\hline $\begin{array}{c}\text { Noise } \\
\text { intensity D }\end{array}$ & 5 & 6 & 6.5 & 7 & 7.5 & 8 & 8.5 & 9 \\
\hline & 24 & 27 & 18 & 27 & 29 & 30 & 33 & 40 \\
& 20 & 28 & 25 & 29 & 35 & 32 & 35 & 24 \\
& 27 & 17 & 28 & 27 & 27 & 25 & 23 & 32 \\
Outlier & 22 & 32 & 32 & 25 & 25 & 32 & 30 & 31 \\
detection in & 27 & 29 & 32 & 36 & 21 & 20 & 23 & 40 \\
every time & 29 & 22 & 24 & 28 & 23 & 24 & 28 & 38 \\
& 25 & 21 & 22 & 24 & 39 & 32 & 41 & 32 \\
& 30 & 33 & 29 & 26 & 28 & 25 & 29 & 32 \\
& 25 & 25 & 30 & 27 & 29 & 32 & 31 & 20 \\
& 32 & 29 & 25 & 27 & 25 & 37 & 31 & 27 \\
\hline Total outlier & 261 & 263 & 265 & 276 & 281 & 289 & 304 & 316 \\
\hline Average value & 26.1 & 26.3 & 26.5 & 27.6 & 28.1 & 28.9 & 30.4 & 31.6 \\
\hline \multirow{2}{*}{ SOH valuation } & 92.9 & 92.5 & 92.1 & 90.1 & 89.3 & 88.1 & 86.3 & 85.1 \\
& $\%$ & $\%$ & $\%$ & $\%$ & $\%$ & $\%$ & $\%$ & $\%$ \\
\hline
\end{tabular}
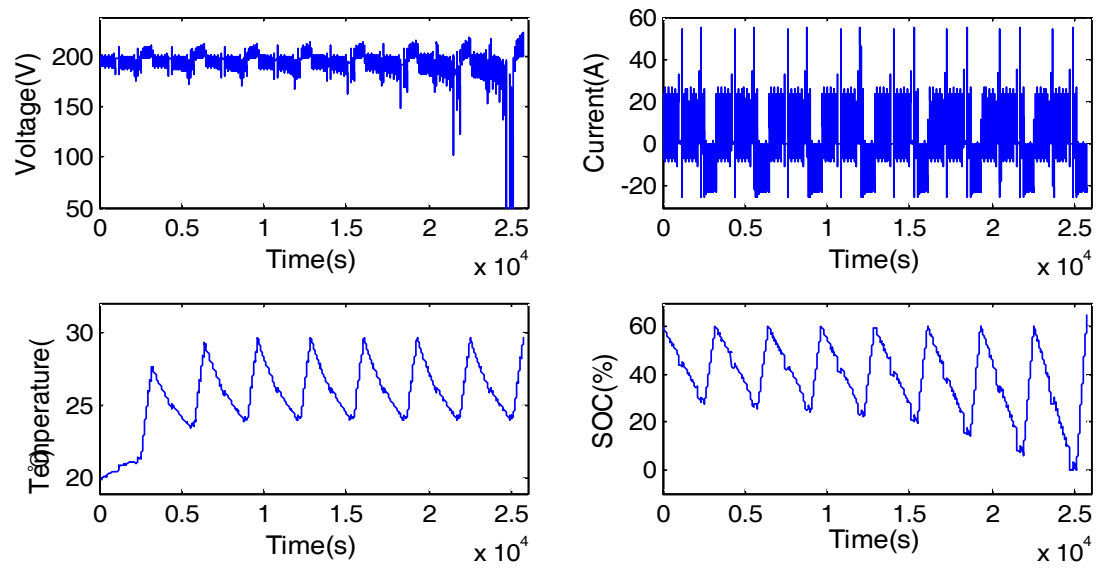

Fig. 9. The migration diagram of battery characteristic parameters under $4 * E C E+E U D C$
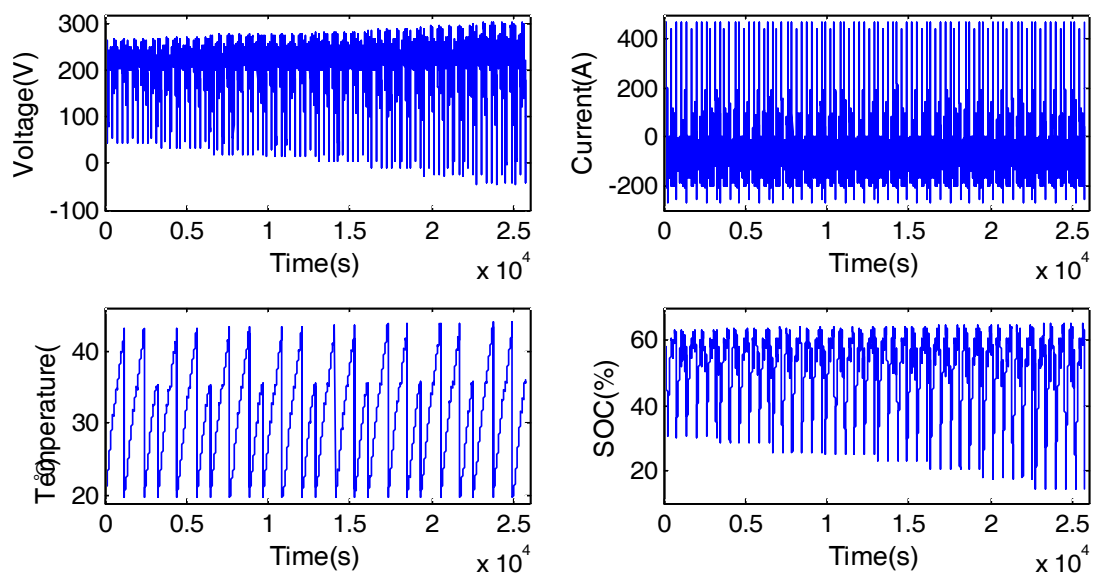

Fig. 10. The migration diagram of battery characteristic parameters under FTP75

Note: while the battery aging, the four parameters (voltage, current, SOC, temperature) change as shown in Fig. 9 and Fig. 10.

\section{Simulation and Analyze}

\subsection{Battery lifetime data}

As indicated in section 3.1, this article selects the battery voltage (Volt), current (Amp), temperature (Temp), and state of charge (SOC) as the judgment for battery SOH. So the data set is composed of the above four parameters. In section 3.4, the parameters of the battery system model in the simulation platform need to be initialized. Combining with practice, the rated capacity initialized as $6.5 \mathrm{Ah}$, 
internal resistance initialized as $0.28 \Omega$, and initial SOC initialized as $60 \%$ and assuming that the temperature of current environmental is 20 degrees. The input of the vehicle simulation platform is the standard driving condition. In this article, 4*ECE+EUDC and FTP75 conditions are applied to be the test conditions.

The following is the process of obtaining the characteristic parameters (In order to the rapid acquisition of data, the simulation environment, compared to the real battery $\mathrm{SOH}$ decreases fast, but it does not affect the validity of the proposed model and the reliability of the data.): Rated capacity is changed with $\mathrm{SOH}$, and their values are shown in Fig. 8.

The simulation model works under the conditions of $4 *$ ECE+EUDC and FTP75. The SOH dropped 5\% after each cycle from $100 \%$ until it reaches $50 \%$. The data was collected every $0.1 \mathrm{~s}$. The total time was $25776 \mathrm{~s}$, so, 257760 data was obtained in all. And these source data formed the test data set $\mathrm{X}$. The test data set $\mathrm{X}$ under 4*ECE+EUDC and FTP75 conditions are shown in Fig. 9 and Fig. 10.

\subsection{Test results and analysis}

As shown in Fig. 11, a graphical user interface (GUI) was established to investigate the process result and the algorithm operation expediently. By using the Windows platform with $2 \mathrm{GHz}$ Intel processor and 2GB memory, the running time was less than $17856 \mathrm{~s}$.

As shown in Fig. 11, click 'START', 'PAUSE' and 'CONTINUE' respectively to correspond to program starting, pausing and going on. In Fig. 11, the left table

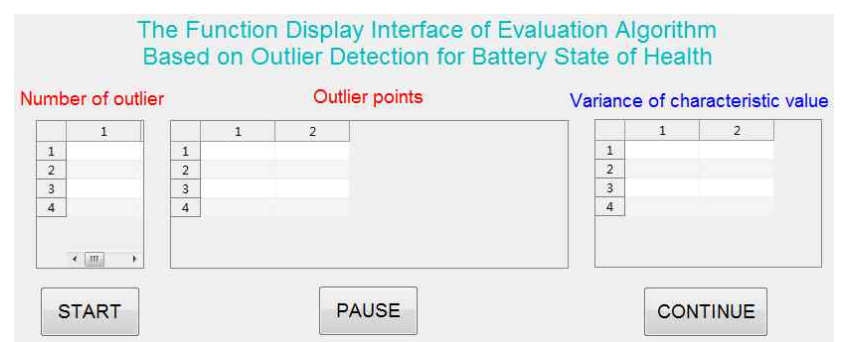

Fig. 11. The GUI of battery SOH estimation algorithm based on outlier detection

Table 3. The average outliers number under different battery $\mathrm{SOH}$

\begin{tabular}{c|c|c}
\hline SOH & $\begin{array}{c}\text { Outlier number } \\
(4 * \text { ECE+EUDC) }\end{array}$ & $\begin{array}{c}\text { Outlier number } \\
(\text { FTP75) }\end{array}$ \\
\hline $100 \%$ & 23.73 & 34.55 \\
$95 \%$ & 25.45 & 43.83 \\
$90 \%$ & 26.40 & 45.20 \\
$85 \%$ & 31.73 & 74.27 \\
$80 \%$ & 44 & 81.36 \\
$70 \%$ & 50 & 87.09 \\
$60 \%$ & 51.1 & 170.30 \\
$50 \%$ & 55.5 & 165.50 \\
\hline
\end{tabular}

(called Table 1) updates once every 300 seconds. The middle table (called Table 2) displays the variance VOA of every characteristic parameter within 300 seconds. By this way, the system directly displays the number of outlier points and other information every 300 seconds.

Under the $4 *$ ECE+EUDC and FTP75 standard driving condition, the number of outliers in different rated capacity and internal resistance is counted, and the Fig. 12 shows the statistical graph of the number of outliers corresponding to every 300 seconds.

The battery system in health condition itself has existed outliers, which contains the system error, system control error (excessive demand of system control for energy led battery system failure at the minor level) and so on. As outlier's number is very little and the data obtained from the vehicle models have been filtered, these outliers will have no interference to the algorithm.

According to the above results, calculate the average number of the outliers under each battery state of health, and the statistical results are shown in Table 3.

According to the results shown in Table 3, the polynomial fitting method was applied to get the fitting equation between the average number of outliers and battery $\mathrm{SOH}$. The Eq. (5) and (6) show the relationship between outlier number and battery SOH in the process of battery lifetime fading. The battery SOH dropped from $100 \%$ to $80 \%$ in $4 * \mathrm{ECE}+\mathrm{EUDC}$ and FTP75 standard conditions.
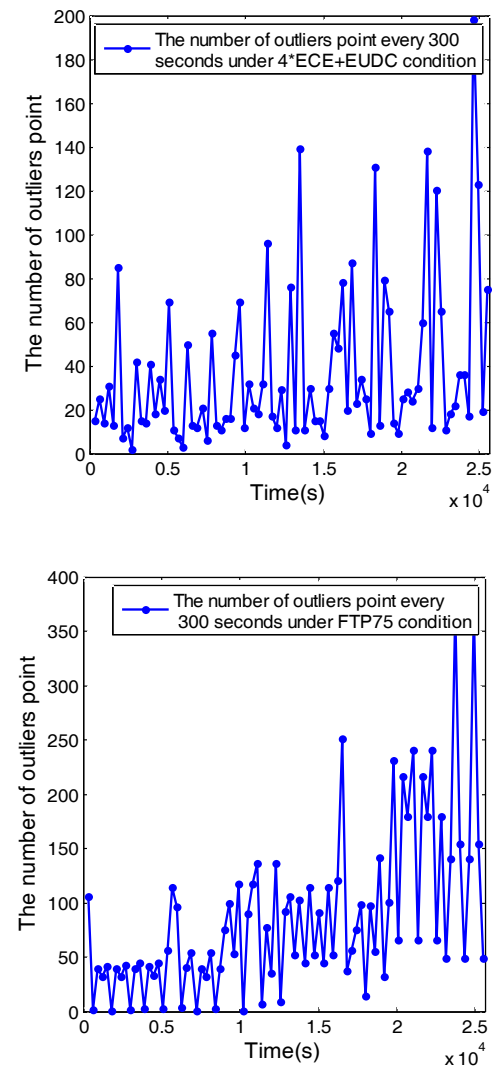

Fig. 12. The statistical graph of the outlier number under two conditions 


$$
\begin{aligned}
S O H= & -0.00455 \cdot x^{3}+0.511 \cdot x^{2} \\
& -19.51 \cdot x+335 \\
S O H= & 0.001673 \cdot x^{2}-0.6309 \cdot x \\
& +118.7
\end{aligned}
$$

In the process of $\mathrm{SOH}$ fading from $100 \%$ to $50 \%$, the comparison graph of the test results under two conditions and polynomial fitting results is shown Fig. 13.

In order to verify the feasibility and effectiveness of the proposed battery $\mathrm{SOH}$ estimation algorithm, based on the HEV simulation software, new historical lifetime charac-
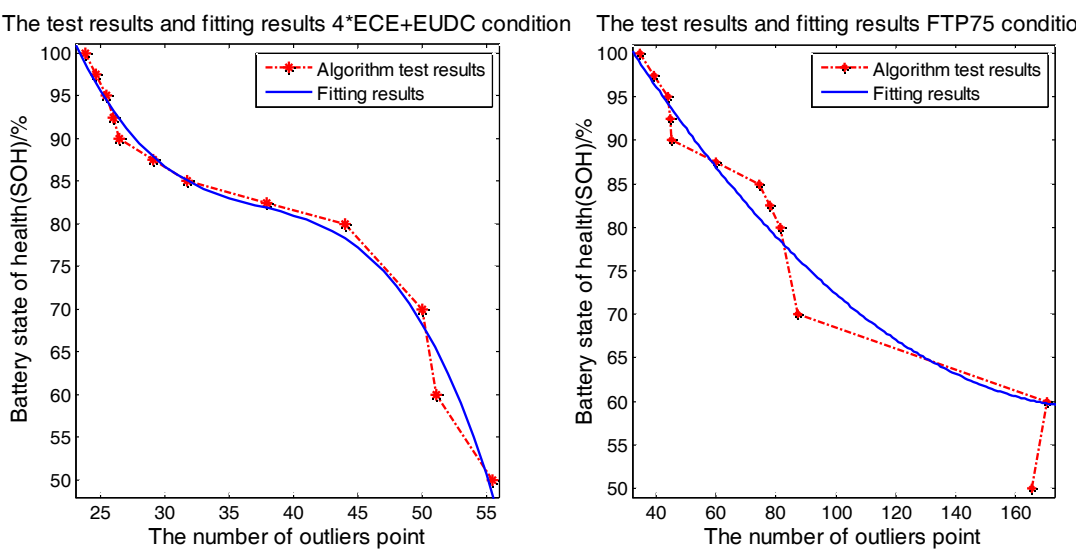

Fig. 13.The fitting and algorithm test results in the process of $\mathrm{SOH}$ fading
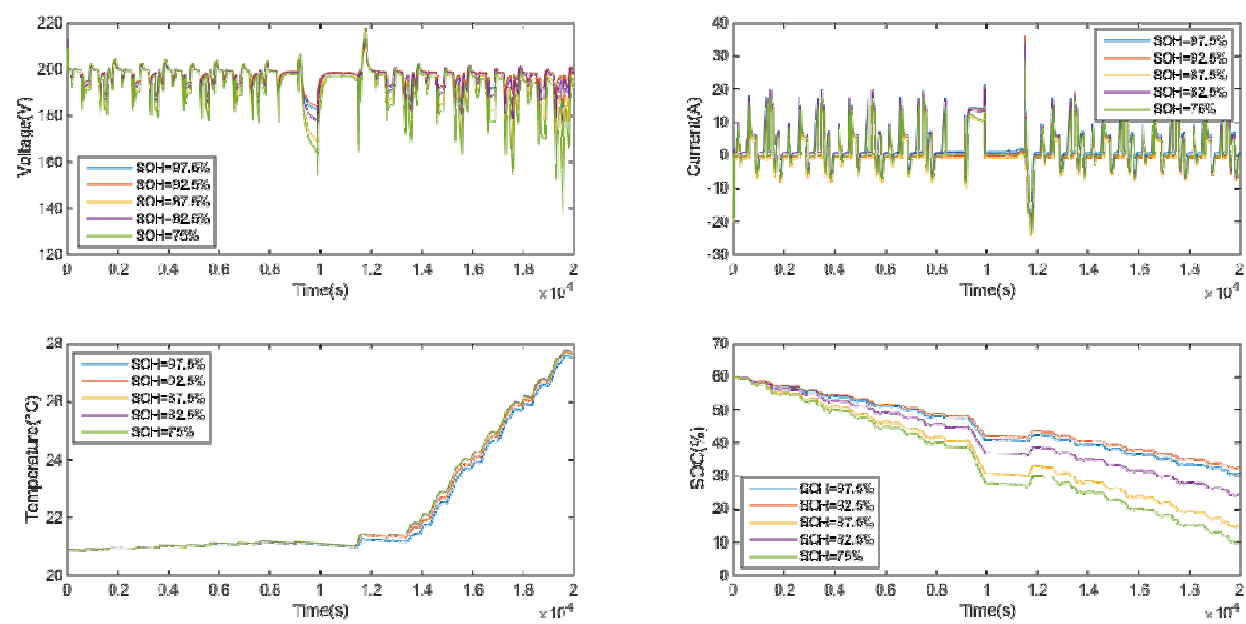

Fig. 14. The battery characteristic parameters under $4 * E C E+E U D C$
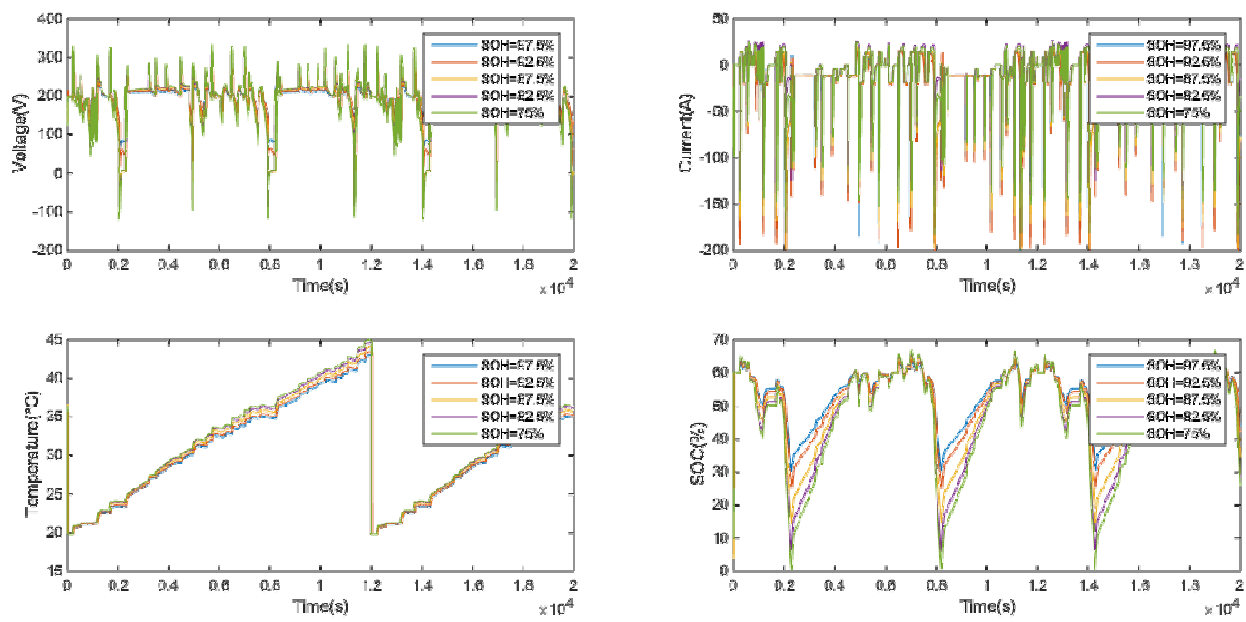

Fig. 15. The battery characteristic parameters under FTP75 
teristic parameters were gained under different standard condition to test and verify the proposed algorithm.

In $4 * \mathrm{ECE}+\mathrm{EUDC}$ and FTP75 standard conditions, the parameter sets of 5 different SOH $(97.5 \%, 92.5 \%, 87.5 \%$, $82.5 \%, 75 \%$ ) were obtained, and the parameter sets are shown in following Fig. 14. and Fig. 15.

As shown in Fig. 14 and Fig. 15, the lower the $\mathrm{SOH}$ is, the greater the parameter fluctuation is, such that with the decrease of SOH the internal resistance has increased, and voltage will has the larger amplitude at the same charge-discharge current, then there is more electric power loss(by thermal power) in charge-discharge process. Therefore, the SOC increased more slowly in charging process, and the SOC decreased faster in discharging process. The fluctuation of the parameters become larger, resulting in more parameters are detected as outliers. With the decrease of $\mathrm{SOH}$, the internal resistance has increased and lead to the open-circuit voltage decreasing. And the current will become smaller under the same load as shown in Fig. 14 and Fig. 15 the fluctuation of the Current has become smaller, so the current does not affect the number of outliers significantly.

The battery characteristic parameters data set are taken into outlier detection by proposed outlier detection algorithm, to obtain the value of the average number of outliers in each state, and the outlier statistics in $4 * \mathrm{ECE}+$ EUDC condition and FTP75 conditions results are shown as Table 4 .

The average outliers number in Table 4 are brought into the fitting function (5) and (6), then the $\mathrm{SOH}$ value of the new state is obtained, and the absolute error and relative error of the $\mathrm{SOH}$ value are calculated. The results are shown in Table 5.

By comparing the true $\mathrm{SOH}$ value with the estimated value, under different conditions, the proposed battery $\mathrm{SOH}$ estimation algorithm based on outlier detection can make an accurate assessment of battery state of health. The

Table 4. The average outliers number under different condition and battery SOH

\begin{tabular}{c|c|c}
\hline SOH & $\begin{array}{c}\text { Outlier number } \\
(4 * \text { ECE+EUDC) }\end{array}$ & $\begin{array}{c}\text { Outlier number } \\
(\text { FTP75) }\end{array}$ \\
\hline $97.5 \%$ & 24.59 & 39.19 \\
$92.5 \%$ & 25.93 & 44.52 \\
$87.5 \%$ & 29.07 & 59.74 \\
$82.5 \%$ & 37.87 & 77.82 \\
$75 \%$ & 47 & 84.82 \\
\hline
\end{tabular}

Table 5. SOH evaluation results and error analysis

\begin{tabular}{c|c|c|c|c|c|c}
\hline \multirow{2}{*}{$\begin{array}{c}\text { Condi- } \\
\text { tion }\end{array}$} & \multicolumn{3}{|c|}{$4 *$ ECE+EUDC } & \multicolumn{3}{c}{ FTP75 } \\
\cline { 2 - 7 } $\begin{array}{c}\text { Ture } \\
\text { SOH }\end{array}$ & $\begin{array}{c}\text { SOH } \\
\text { evaluation }\end{array}$ & $\begin{array}{c}\text { absolute } \\
\text { error }\end{array}$ & $\begin{array}{c}\text { relative } \\
\text { error }\end{array}$ & $\begin{array}{c}\text { SOH } \\
\text { evaluation }\end{array}$ & $\begin{array}{c}\text { absolut } \\
\text { e error }\end{array}$ & $\begin{array}{c}\text { relative } \\
\text { error }\end{array}$ \\
\hline $97.5 \%$ & $96.58 \%$ & $-0.92 \%$ & $-0.94 \%$ & $96.54 \%$ & $-0.96 \%$ & $-0.98 \%$ \\
\hline $92.5 \%$ & $93.36 \%$ & $0.86 \%$ & $0.93 \%$ & $93.83 \%$ & $1.33 \%$ & $1.44 \%$ \\
\hline $87.5 \%$ & $87.90 \%$ & $0.40 \%$ & $0.45 \%$ & $86.98 \%$ & $-0.52 \%$ & $-0.59 \%$ \\
\hline $82.5 \%$ & $81.89 \%$ & $-0.61 \%$ & $-0.74 \%$ & $79.74 \%$ & $-2.77 \%$ & $-3.35 \%$ \\
\hline $75 \%$ & $74.43 \%$ & $-0.57 \%$ & $-0.75 \%$ & $77.22 \%$ & $2.22 \%$ & $2.96 \%$ \\
\hline
\end{tabular}

absolute error under $4 * \mathrm{ECE}+\mathrm{EUDC}$ condition is less than $1 \%$, and the absolute error under FTP75 condition is less than $3 \%$. So, the equations (5) and (6) can represent the relationship between outlier number and $\mathrm{SOH}$ accurately. The outlier detection algorithm succeeds in evaluating battery $\mathrm{SOH}$.

\section{Conclusion}

The test results show that the proposed outlier detection algorithm can recognize the outlier points accurately under different conditions. The number of outlier points in the outlier data set increases gradually with the battery $\mathrm{SOH}$ fading. And the fitting function established in this paper can reflect the relationship between battery $\mathrm{SOH}$ with the current outlier data distribution, and the absolute error of the evaluation results and the real value of $\mathrm{SOH}$ is less than $3 \%$. The evaluation algorithm based on outlier detection for battery state of health can estimate the battery $\mathrm{SOH}$ in real time accurately.

\section{Acknowledgments}

This work is supported by CQ CSTC (cstc2015zdcyztzx60006, cstc2014jcyjA60004, cstc2014jcyjA1919).

\section{References}

[1] Hannan, M.A, et al, "Hybrid electric vehicles and their challenges: A review," Renewable \& Sustainable Energy Reviews, vol. 29, no. 7, pp. 135-150, 2014.

[2] Chen Ping, Piao Changhao, et al, "Modeling of battery management system software in virtual simulation environment," Journal of Automotive Safety and Energy, vol.4, no.1, pp.67-74, 2013.

[3] Ying Xie, Zhao-guang Wang, et al. "Development of Battery System Testing Machine," International Journal of Future Engineering, vol.9, no.5, pp. 1-7, 2015.

[4] Changhao Piao, Wenli Fu, Chongdu Cho, et al, "Online Parameter Estimation of the Ni-MH Batteries Based on Statistical Methods," Energies, vol.3, no.2, pp. 206-215, 2010.

[5] Huet F, "A review of impedance Measurements for Determination of the state-of-charge and state-ofhealth of Secondary Batteries," Journal of Power Sources, vol.70, no.1, pp. 59-69, 1998.

[6] Calabek M, Micka K, et al, "Resistance Changes and Premature Capacity Loss in Lead Battery Plates," Journal of Power Sources, vol.62, no.2, pp. 161-166, 1996.

[7] Fan J, Fedkiw P, "Electrochemical impedance spectra 
of full cells: relation to capacity and capacity-rate of rechargeable Li cells using $\mathrm{LiCoO} 2, \mathrm{LiMn} 2 \mathrm{O} 4$, and LiNiO2 cathodes," Journal of Power Sources, vol.72, no.2, pp. 165-173, 1998.

[8] Gregory L, Plett, "Extended kalman filtering for battery management systems of LiPB-based HEV battery packs, part 3. state and parameter estimation," Journal of Power Sources, vol. 134, pp. 277-292, 2004.

[9] Dai Haifeng, Sun Zechang, "Estimation of Internal States of Power Lithium-ion Batteries Used on Electric Vehicles by Dual Extended Kalman Filter," Journal of Mechanical Engineering, vol. 45, no. 6, pp. 95-101, 2009.

[10] H. Nakamura, D. Yumoto, "The application of adaptive digital filter for the internal state estimate on of batteries," SCIE Annual Conference in Fukui, Fukui, Japan, 2003.

[11] IL-Song Kim, "A technique for estimating the state of health of lithium batteries through a dual-slidingmode observer," IEEE Transactions on Power Electronics, vol. 25, no. 4, pp. 1013-1022, 2010.

[12] Edwin M, Raymond T, "Algorithms for Mining Distance-Based Ouliers in Large Datasets," Very Large Data Bases Conference Proceedings, University of Vienna, Austria, 1998.

[13] Piao Changhao, Huang Zhi, "High-Dimensional Data Stream Outlier Detection Algorithm Based on Angle Distribution," Journal of Shanghai Jiao Tong University, vol. 48, no. 5, pp. 647-652, 2014.

[14] Kriegel H P, Schubert M, Zimek A, "Angle-based outlier detection in high dimensional data," Proceedings of the 14th ACM SIGKDD International Conference on Knowledge Discovery and Data Mining, New York, USA, 2008.

[15] Pham N, Pagh R, "A near-linear time approximation algorithm for angle-based outlier detection in high dimensional data," Proceedings of the 18th ACM SIGKDD International Conference on Knowledge Discovery and Data Mining, New York, USA, 2012.

[16] Changhao Piao, Huang Zhi, Ling Su and Sheng Lu, Research on Outlier Detection Algorithm for Evaluation of Battery System Safety, Advances in Mechanical Engineering, vol. 6, pp. 1-7, January 2014

[17] Lu L, Han X, Li J, et al. "A review on the key issues for lithium-ion battery management in electric vehicle," Journal of Power Sources, vol. 226, no. 6, pp. 272-288, 2013.

[18] Wang J, Liu P, Jocelyn H G, et al. "Cycle-Life Model for Graphite-LiFePO4 Cells," Journal of Power Sources, vol.196, pp. 3924-3948, 2011.

[19] Chang-hao Piao, Qi-fan Yu, et al. "Virtual Environment Modeling for Battery Management System," Journal of Electrical Engineering \& Technology, vol. 9, no. 5, pp. 1729-1738, 2014.

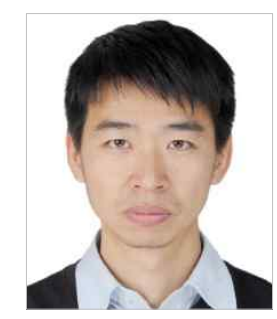

Changhao Piao was born in Yanbian, China in 1978. He is a professor in Chongqing University of Posts and Telecommunication. He received his B.S. degree from Xi'an Jiaotong University, China in 2004. He obtained his Ph.D. in Inha University, Korea. His primary research interests are new energy vehicle systems, active safety systems, and artificial intelligence.

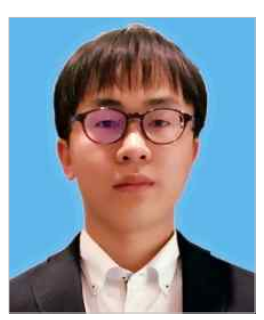

Zihao Hu was born in Hubei, China in 1992. He is a postgraduate researcher in the Department of Pattern Recognition and Application at Chongqing University of Posts and Telecommunication. His research focus is battery management system and power battery technology.

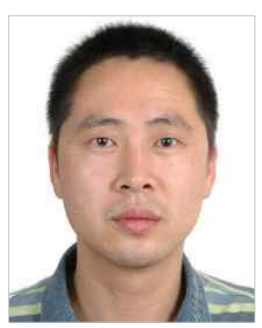

Ling Su was born in 1975 in Chongqing (China). He received his BS degree from Xi'an Jiaotong University, China in 1998 and obtained Ph.D. also from Xi'an Jiaotong University in 2005. $\mathrm{He}$ is now a deputy chief engineer in Chongqing changan new energy automobile co., LTD., His primary research interests are in new energy vehicles and its control system.

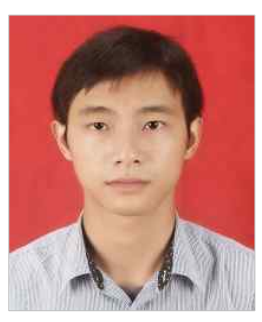

Jianfei Zhao was born in 1989 in Chongqing (China). He is a Postgraduate Researcher in the department of Pattern Recognition and Application at the Chongqing University of Posts and Telecommunications. His research focus is battery management system and power battery technology. 